\title{
Towards an Universal Method for Predicting Eddy-Current Sensor Characteristics in the Railway Industry
}

\author{
Marek Augustyniak $^{1,2}$ (D) Piotr Borzyszkowski ${ }^{2} \cdot$ Mariusz Buława $^{3}$
}

Received: 11 June 2018 / Accepted: 19 February 2019 / Published online: 2 March 2019

(c) The Author(s) 2019

\begin{abstract}
Eddy current (EC) sensors placed in the vicinity of a rail track and sensitive to passing train components are safety-critical components commonly used in the railway sector. From the engineering point of view they form a system with numerous variables including their geometry, the lift-off, magnetic properties of the applied materials, the operating frequency and electrical characteristics of the built-in circuits. Although some simple configurations of the EC devices have been studied experimentally, numerically and analytically, there have so far been no universal algorithm allowing for predicting, understanding and optimizing output signals for arbitrarily device/track/wheel characteristics. A significant step towards such an universal algorithm is presented in this work, combining a linear 3D finite element modelling and a set of analytical formulas derived directly from the constants of the resonance circuit. Functions correlating the magnetic induction space/time characteristics with the circuit outputs are proposed and validated against experiment in three stages: (a) by determination of the signal from a flat thin FeSi plate (i.e. in fully controlled laboratory conditions), (b) by laboratory measurements of a highly degraded rail with uncertain material properties, (c) by tests of a commercial EC wheel detector, with reference to averaged service data. The proposed methodology can be extended to any NDT field where EC devices are applied.
\end{abstract}

Keywords Eddy-current testing $\cdot$ Electromagnetic axle counters $\cdot$ Finite element modelling $\cdot$ Resonant circuits

\section{Introduction}

The eddy-current (EC) based sensors are widely used in Nondestructive Testing in the railway industry. EC defect detectors, as well as electromagnetic axle counters (a.k.a. "wheel detectors" or "train wheel sensors") are safetycritical devices which require a thorough understanding in order to correctly interpret noisy signals and avoid both false-positive and false-negative indications. However, the complexity of the problem is such, that in sensor-producing companies known to the authors the empirical trial-and-error approach tends to prevail over comprehensive theoretical insight. Even if undisturbed by noise sources, the EC sensor output depends on numerous factors, such as the coil

Marek Augustyniak

maugustyniak@mif.pg.gda.pl

1 Dept. of Applied Physics, Gdansk University of Technology, Gdańsk, Poland

2 DES ART Ltd, Gdynia, Poland

3 VOESTALPINE Signalling, Sopot, Poland geometry, circuit configuration, operating frequency, shape of the measured object, its electromagnetic properties (possibly nonhomogeneous and nonlinear), and presence of metallic auxiliary parts within the device. The surface condition and material properties have also an influence on the EC measurement result.

The EC NDT devices with resonant RLC circuits have been devoted numerous publications, including but not limited to the railway context. Some representative examples are mentioned below. Several authors have examined the laboratory or in situ the properties of the excitation coil(s) and the entire device, determining the sensitivity to the material defect, lift-off influence, or proposing better signal analysis schemes (e.g. [1-5]). The purely experimental and statistical approach does not, however, provide insight into underlying phenomena. In particular, it does not determine the relationship between the magnetic field distribution and the output signal dynamics.

Another approach consists in numerical [usually finite element analysis (FEA)] reconstruction of the amplitudes and phases of magnetic fields and ECs within the target object and in the surrounding space. The modelling is 
usually accompanied by experimental validation and aims at guiding the further development of the EC NDT methodology. A representative modelling and experimental work by Rocha et al. [6] focuses on performance of magnetic probes tested in velocity EC testing. In another research [7], Zhu et al. model a rail with a non-homogeneous magnetic permeability distribution, at excitation frequencies between $1 \mathrm{~Hz}$ and $100 \mathrm{kHz}$. A similar numerical and experimental work concerning an EC sensor over a layered material has been performed by Augustyniak et al. [8]. These works allowed for physical interpretation of some key phenomena, such as e.g. "zero-crossing" frequencies due to the opposing influence of the conductivity and magnetisation, but they referred to a single, relatively simple configuration of the detection device and could not be easily generalised.

Only few researchers have aimed at constructing purely or dominantly analytical formulas capable of predicting and explaining the EC sensor output in the vicinity of a metal object. Among them, Kim et al. [9] introduce an equivalent network circuit representing the EC sensor and the ECs generated withing a metal plate. They start from the Biot-Savart's formula, allowing determination of mutual inductance between the sensors' coil and the EC flowing in the target object. The approach assumes, that the target object is flat, and axially symmetrical with regard to the sensor, which is rarely the case in the railway context, except for the examination of the separate axle or the wheel's circumference. In another paper [10], Enoki et al. tackle the problem of predicting the resistance and inductivity variation of coils situated in the vicinity of a rail, at a high frequency typical for some railway sensors $(450 \mathrm{kHz})$. They develop a closed-form dependency of the electrotechnical parameters (resistance R, inductance L) on coil's distance from the rail head top surface. Enoki's approach has mixed analytical/ empirical nature, which limits its application to a narrow set of rail/sensor configurations. Yet more importantly, it does not derive the results from the fundamental parameter, which is the space and time distribution of the magnetic induction. Instead, the skin effect and the associated field distribution are implicitly involved in a set of intermediate empirical parameters. Finally, Klein et al. [11] has recently defined a semi-analytical model of the EC response of a coil operating inside two conducting concentric tubes. The excellent agreement was found between the model and the reference (numerical and experimental) data, but still the approach remains limited to a precisely defined sensor/ object configuration.

To sum up this brief literature review focusing on the special area of axle counters, the experimental observations and advanced signal processing tend to be separated from theoretical background and magnetic field modelling, while the analytical approaches-useful as they are-can not be generalised to complex cases typical for industrial practice. More importantly, to the best authors' knowledge, there have been no published attempts to precisely define a relationship between the magnetic field and electrotechnical variables in the railway EC sensor context.

Our approach aims at combining numerical and analytical strategies of representing the EC sensor set-up, providing the key link between the magnetic field and the behaviour of the circuit, for an arbitrary rail/wheel/ coil configuration, with as little calibration and empirical parameters as possible.

The approach starts from the fundamental observation that both the effective resistance $\mathrm{R}$ and inductivity $\mathrm{L}$ of the measuring coil are dependent on the presence of a metallic object. Starting from electrotechnical formulas relevant to either a serial or parallel resonant circuit, we postulate a relatively simple association between effective values of $(\mathrm{R}, \mathrm{L})$, and the amplitude/phase of the magnetic induction within the coil, which in turn can be determined from a relatively straightforward FEA. This is a significant step towards prediction of the output signal of any coil-based sensor in the vicinity of an arbitrarily shaped rail and wheel, and such an approach is potentially extensible to other areas of application of EC-based devices.

It is worth emphasising again, that the idea of incorporating electromagnetic FEA into the process of developing NDT devices (including EC-based ones) and gaining deeper insight into the underlying phenomena if not new. A recent review of the field has been proposed in [12]. Many of the available works include detailed investigation of nonlinearities associated with ECs $[13,14]$, as well as improved FE formulations [13, 15], subtle micro-magnetomechanics [14] or consideration of technological process like press hardening or hot stamping of examined object [16]. The works of Yasmina Gabi and her co-authors are particularly similar to our strategy, in terms of combination of experimental data, numerical model and analytical formulas for the sake of NDT system calibration and enhancement. However, our work places distinctly different accents. Its main distinctive contributions are:

- focusing on the transition between electromagnetic fields and the circuit electrodynamics, and proposing an original, physics-based coupling formula, which allows for exhaustive explanation of experimental phenomena used in the commercial axle counters,

- tacking the high-frequency domain (as compared with $50 \mathrm{~Hz}$ or $200 \mathrm{~Hz}$ in the aforementioned articles), where capturing skin effects with appropriate mesh size and shape is a difficult task,

- multi-stage validation involving simple lab sample, then a short rail track segment, and finally a study and successful reproduction of service data of the actual measuring devices. 
On the other hand, we have decided not to propose modifications of standard FE algorithms, and precise determination of material data (including nonlinearities) has been given secondary attention.

\section{Basic Version of the Field-Circuit Coupling Function}

The formulas developed below refer to a serial RLC circuit with a negligible capacitance and a coreless coil placed far from any metal object. Its complex impedance can be written as:

$\widetilde{Z_{0}}=R_{0}+j \omega L_{0}$.

If the inductor (i.e. the coil or a set of coils) is moved near some conducting object, the inductance changes its amplitude and becomes complex:

$\widetilde{Z_{1}}=R_{0}+j \omega \tilde{L_{1}}$.

It is postulated, that the complex inductance can be decomposed into two following parts, producing:

$\widetilde{L_{1}}=\left(L_{0}+L_{S H}\right)+j L_{E D}$

here $\mathrm{L}_{\mathrm{SH}}$ results from the redistribution of the magnetic flux lines which are shielded by the metal object, and contributes to the "normal" part of the inductance, i.e. the one which exhibits a $90^{\circ}$ phase lag with regard to the source current. Apart from the deformation of flux lines, the activity of ECs in the metal object entails a phase shift in the magnetic flux with respect to the magnetising current, and generates the "anomalous" component of the coils' inductance, noted as $\mathrm{L}_{\mathrm{ED}}$, being "in-phase" with the source current.

Consequently there is a cross-dependence of the real and imaginary components of the magnetic flux and the coil's inductance, i.e.:

- the purely real characteristics of the magnetic flux causes a "normal" inductance of the coil, influencing uniquely the imaginary component of the circuit's impedance,

- any imaginary component of magnetic flux generates an "anomalous" contribution to the inductance, disturbing the real component of circuit's impedance; this fact is fundamental for signal generation and interpretation in EC NDT.

Combination of (2) and (3) leads to the observation, that the "anomalous" inductivity $\mathrm{L}_{\mathrm{ED}}$ modifies the circuit's effective resistivity:

$\widetilde{Z_{1}}=\left(R_{0}-\operatorname{Im} \tilde{L_{1}}\right)+j \omega\left(\operatorname{Re} \tilde{L_{1}}\right)$

which in turn can be written as:

$\widetilde{Z_{1}}=\left(R_{0}-\omega L_{E D}\right)+j \omega\left(L_{0}+L_{S H}\right)$.
The component $-\omega \mathrm{L}_{\mathrm{ED}}$ can also be termed $\mathrm{R}_{\mathrm{ED}}$, and represents the EC related contribution to the effective resistance of the circuit.

The resistance and inductance measurable directly at the circuit's output can be thus written as:

$R_{1}=\left(R_{0}-\omega L_{E D}\right)$,

$L_{1(\text { norm })}=\left(L_{0}+L_{S H}\right)$.

The $\mathrm{L}_{\mathrm{ED}}$ and $\mathrm{L}_{\mathrm{SH}}$ parameters can be then deduced from experimental data as:

$L_{E D}=\left(\frac{1}{\omega}\right)\left(R_{0}-R_{1}\right)$,

$L_{S H}=L_{1(\text { norm })}-L_{0}$.

So far, only electrotechnical variables have been taken into account, but no reference to the magnetic field has been made. The quantitative link between the circuits' effective inductance and the magnetic flux within the coil is defined in the following formulas. First, the average magnetic induction normal to the coil's cross-section can be written in a complex form as:

$\tilde{B}=B_{\mathrm{Re}}+j B_{\mathrm{Im}}$.

Starting from the standard, non-complex definition of coils' inductance (voltage proportionality to the time variation of current), we have extended it to a complex form, and assumed a proportionality between average magnetic field within the coil's cross section and the instantaneous current (both written again in the complex form). Based on these premises, it is postulated, that for any coil placed in a harmonically varying magnetic induction the components of its inductance fulfill the relationship:

$\frac{\operatorname{Im} \tilde{L}}{\operatorname{Re} \tilde{L}}=\frac{B_{\mathrm{Im}}}{B_{\mathrm{Re}}}$.

which, after application of (3), leads to

$\frac{L_{E D}}{L_{0}+L_{S H}}=\frac{B_{\mathrm{Im}}}{B_{\mathrm{Re}}}$.

This Eq. (10) constitutes a key hypothetical relationship between the AC magnetic field within the sensor coil and the electrotechnical characteristics of a serial resonant circuit. Once validated, the presented set of equations allows for the at least qualitative prediction of the voltage-current function of the sensor coil, which is fundamental for construction and optimization of ECT measurement devices.

Note, that in the following discussion, the left-hand side of Eq. (10) is referred to as "ECAR-L" (inductancerelated), and the right-hand side as "ECAR-B" (fieldrelated), where ECAR stands for "Eddy Current Activity Ratio". 


\section{Case of a FeSi Plate}

\subsection{FeSi Plate: The Measuring Setup}

In order to validate the basic version of the field-circuit coupling function, a fully-controlled laboratory case of a thin flat FeSi plate has been studied.

The impedance analyser HIOKI-3522-5 has been applied to record the following parameters: resistance, inductance, current/voltage phase shift and impedance modulus. The measurements have been performed at $100 \mathrm{kHz}$ (the maximum frequency available for this analyser) with an imposed voltage range of $1 \mathrm{~V}$. Four coaxial cables connected the analyser to a coil, which could either be placed "in the air" or in the vicinity of the studied object (a metal plate or the rail segment). The plate had dimensions: $160 \times 100 \mathrm{~mm}$ and the thickness of $0.35 \mathrm{~mm}$.

Two different coils have been prepared with characteristics given in Table 1. The larger coil designated as X1 was well suited for measurement at the flat FeSi plate, while the smaller coil X2 was apt for measurements in the concave space below the rail's head, as discussed later in this paper.

The X1 coil's lift-off distance in this experiment was constant, set to $1 \mathrm{~mm}$.

\subsection{FeSi Plate: The Finite Element Model}

The FE model of the FeSi plate created in HyperMesh/ ANSYS software, presented in Fig. 1, consisted of 30,000 full-3D, 20-noded SOLID117 elements and 130,000 nodes. For the sake of maximum accuracy, it has been constructed uniquely of hexahedral elements. The mesh within gap between the plate and the bottom edge of the coil was refined, and the plate itself is divided into uniform $\sim 20 \mu \mathrm{m}$-thick element layers, in order to accurately capture the skin effect at $100 \mathrm{kHz}$. A double symmetry plane has been applied in order to minimize computational effort.

The sinusoidally varying current density has been applied to the coil's cross section, resulting in the total current of 1 A-turn.

Table 1 Characteristics of coils used in laboratory tests

Diameter (mm) Axial length (mm) Number
of turns

\begin{tabular}{llll}
\hline Coil X1 & 28 & 10 & 100 \\
Coil X2 & 16 & 10 & 100 \\
\hline
\end{tabular}

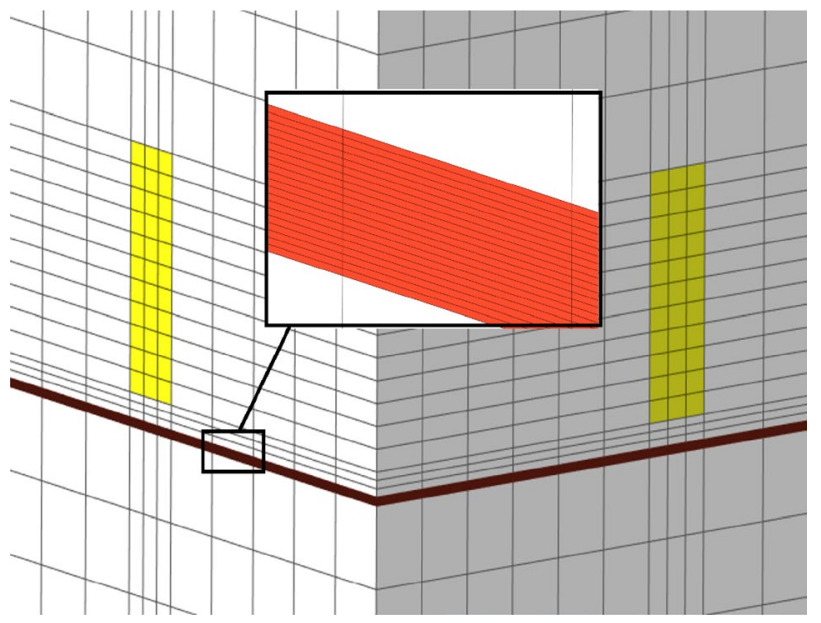

Fig. 1 A quarter-section of the coil (yellow) placed at $1 \mathrm{~mm}$ from the FeSi plate (red) (Color figure online)

The magnetic permeability of the plate has been adopted as 4000 , and its resistivity set as $5 \times 10^{-7} \mathrm{Ohm}$ meter, which are values typical for $3 \%$ Si electrical steel.

The magnetic flux has been forced to be parallel to all the model's boundaries, and the EC time-integrated potential has been coupled at all the nodes belonging to the metal plate and lying on the symmetry planes, in order to assure the correct current flow at these boundaries.

\subsection{FeSi Plate: Comparison of Experimental and Modelling Results}

A single series of measurements of the coil's resistance and inductance and the corresponding field computation has been performed for the configuration described in the Sect. 3.2, at the excitation frequency of $100 \mathrm{kHz}$.

The ECAR-L parameter deduced from the experiment has been compared against the ECAR-B deduced from simulation. The key data is presented in Table 2:

The correlation between the measured and the simulated ECAR is perfect for the case of well-defined laboratory conditions, including a constant lift-off distance, simple object's shape, and macroscopically flat, polished surface.

The next step towards the validation of the coupling function is studying a non-trivial geometry exhibiting a very rough surface and having uncertain material properties, at various coil's lift-off distances. 
Table 2 Comparison of experimental and numerical results for the FeSi plate test

\begin{tabular}{llll}
\hline Parameters & Symbols & Values & Units \\
\hline Measured resistance "in the air" & $\mathrm{R}_{0}$ & 9.0 & $\Omega$ \\
Measured resistance "by the plate" & $\mathrm{R}_{1}$ & 20.9 & $\Omega$ \\
Measured inductance "in the air" & $\mathrm{L}_{0}$ & 340 & $\mu \mathrm{H}$ \\
Measured inductance "by the plate" & $\mathrm{L}_{1(\text { norm) }}$ & 336 & $\mu \mathrm{H}$ \\
Eddy-current inductance component & $\mathrm{L}_{\mathrm{ED}}$ & -19 & $\mu \mathrm{H}$ \\
Shielding inductance component & $\mathrm{L}_{\mathrm{SH}}$ & 4 & $\mu \mathrm{H}$ \\
Averaged imaginary induction in the coil & $\mathrm{B}_{\mathrm{Im}}$ & -2.47 & $\mu \mathrm{T}$ \\
Averaged real induction in the coil & $\mathrm{B}_{\mathrm{Re}}$ & 43.4 & $\mu \mathrm{T}$ \\
$\begin{array}{c}\text { Eddy current activity ratio deduced from } \\
\text { measurement }\left[\mathrm{L}_{\mathrm{ED}} / \mathrm{L}_{1(\text { norm }}\right]\end{array}$ & $\mathrm{ECAR}_{\mathrm{L}} \mathrm{L}$ & -0.057 & - \\
$\begin{array}{c}\text { Eddy current activity ratio deduced from } \\
\text { simulation }\left(\mathrm{B}_{\mathrm{Im}} / \mathrm{B}_{\mathrm{Re}}\right)\end{array}$ & ECAR-B & -0.057 & - \\
\hline
\end{tabular}

\section{Case of a Narrow-Gauge Rail}

\subsection{Narrow-Gauge Rail: Measuring Setup}

The semi-industrial set-up consisted of a single, $540 \mathrm{~mm}$ long segment of a narrow-gauge rail track, dimensioned as in Fig. 2. The rail segment was acquired from the decomissioned railway track, and no service history or material specification was provided. The measured dimensions did not correspond to any of available standards, perhaps because of significant wear of the rolling surface. The lack of data was unfavourable from the point of view of modelling precision, but beneficial when aiming at reproducing the reality of industrial application of sensors, where precise data is

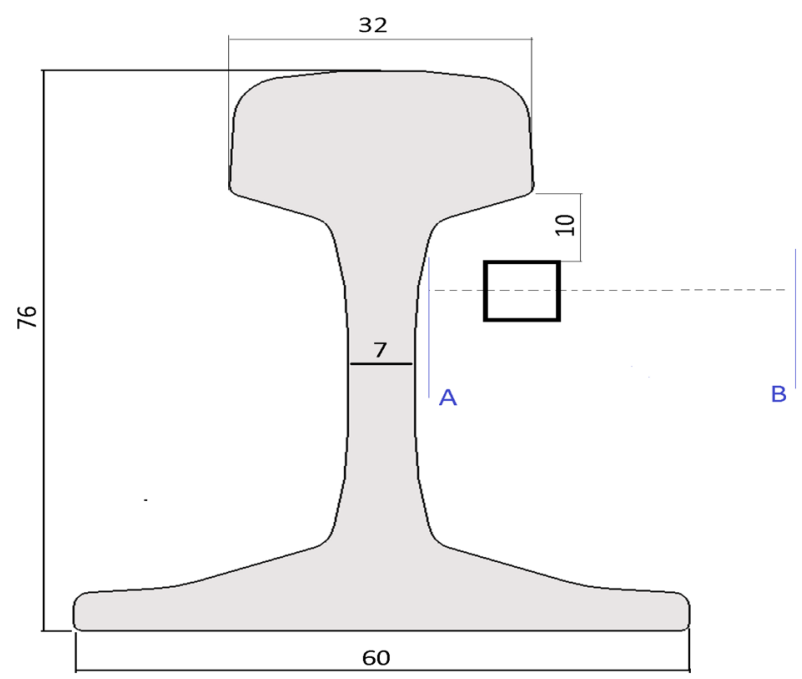

Fig. 2 The cross-section and dimensioning of the narrow-gauge rail track; all units in $(\mathrm{mm})$, vertical lines marked " $\mathrm{A}$ " and "B" indicate the limits of coils' movement scarce as well. The rail surface was left in its initial state, with significant rugosity, as seen in Fig. 3. The "X2"-type coil was placed in the vicinity of the rail, with a possibility of controlling its position along the axis (1-40 mm).

\subsection{Narrow-Gauge Rail: Finite Element Model}

The FE model of the narrow gauge rail track created in HyperMesh/ANSYS software consisted of 600,000 SOLID117 elements and 750,000 nodes. The key regions, including the outer $2 \mathrm{~mm}$ of the rail have been constructed from hexahedral, thin elements, featuring the minimal thickness of $\sim 20 \mu \mathrm{m}$ at the air-metal interface, allowing for accurate representation of the skin effect at $100 \mathrm{kHz}$. A single symmetry plane has been applied, and the half of the rail opposite to the coil has not been modeled because of the negligible magnetic flux penetrating into that area. The model's structure is presented in Figs. 4 and 5.

The magnetic permeability of the rail segment has been adopted as 200, and its resistivity set as $2 \times 10^{-7} \mathrm{Ohm}$ meter. These parameters were collected from [17-19], and accepted as typical for steel grades used for railway tracks.

The magnetic flux has been forced to be parallel to the symmetry plane, and the EC time-integrated potential has been coupled at all the nodes belonging to the rail and lying on the symmetry planes, in order to assure the correct current flow at these boundaries.

The sinusoidally varying current density has been applied to the coil's cross section, resulting in the total current of 1 A-turn.

\subsection{Narrow-Gauge Rail: Comparison of Experimental and Modelling Results}

The validation of the coupling function in the case of a rail track segment was carried out similarly to the case of

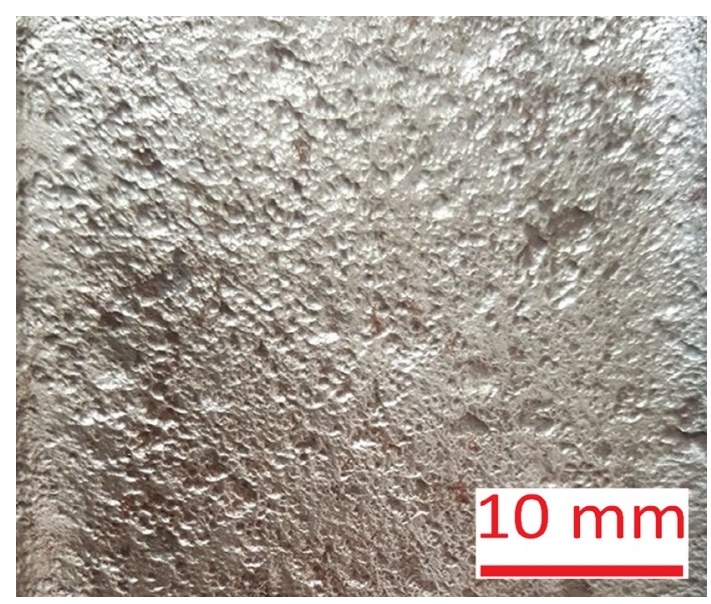

Fig. 3 The rugosity of the rail track's surface used in the experiment 


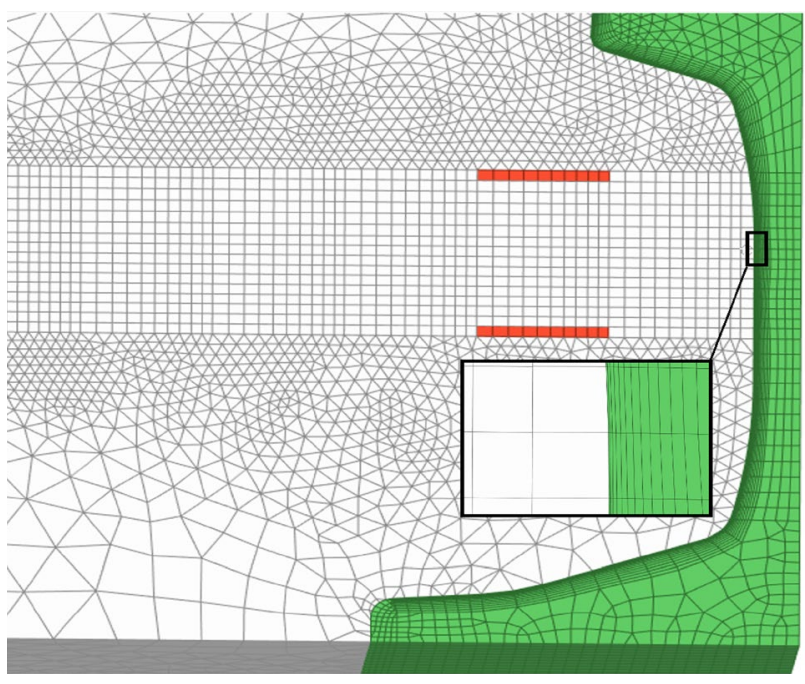

Fig. 4 Cross-section of the narrow-gauge track model

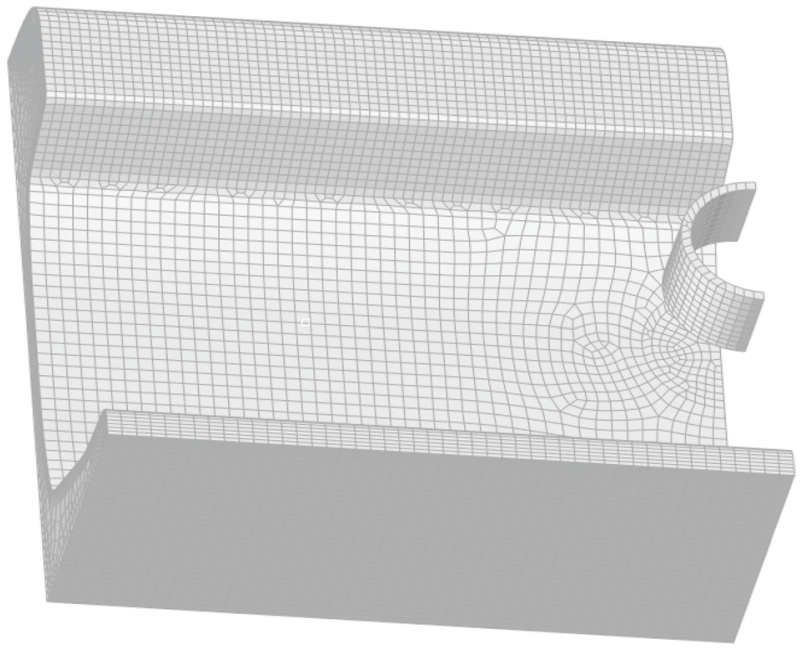

Fig. 5 Isometric view of the model (air elements removed)

a thin FeSi plate. The coil designated as $\mathrm{X} 2$ was used to measure the effective resistance and inductance at varying coil-rail distances.

Although performed in laboratory, the experiment had features resembling in situ conditions, e.g.:

(a) magnetic permeability and electric conductivity of the rail material were unknown and could only be roughly estimated,

(b) the object's surface was pitted and covered with oxide/ protective coating of unknown characteristics,

(c) the set-up was not shielded against external electromagnetic noise.

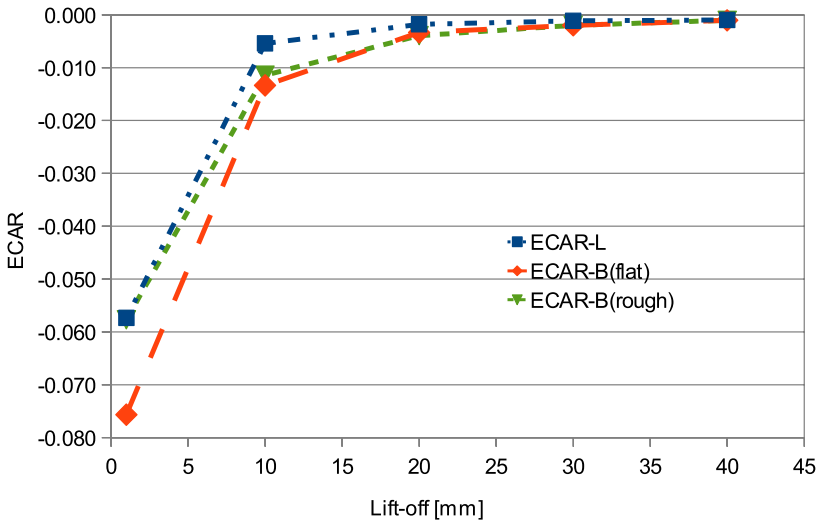

Fig. 6 Narrow-gauge rail test; experimental (-L) versus numerical (-B) results

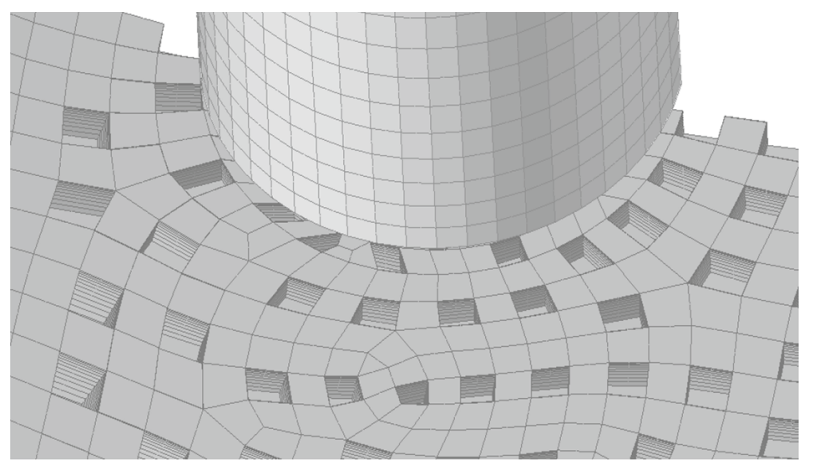

Fig. 7 A simplified method of introducing roughness into the FEA model; the pits are of order of $1 \times 1 \times 1 \mathrm{~mm}$

The values of ECAR-L (experimental) and ECAR-B (numerical) as a function of the lift-off were determined and compared, as seen in Fig. 6. The first attempt to correlate parameters ECAR-L and ECAR-B (series named "rough") showed a qualitative agreement, with a similar exponential trend and negative sign obtained in both the modelling and experiment. The discrepancy between the numerical ECAR$\mathrm{B}$ and experimental ECAR-L was the most distinct at the minimal lift-off distance $(1 \mathrm{~mm})$. This observation could be due to the significant difference between the modelled and actual characteristics of the surface layer of the rail, as the rough surface decreased the effective magnetic permeability of the object, and dampened the activity of ECs, thus influencing the ECAR-L value.

A yet better correlation was found (see Fig. 6, numerical data noted as "flat"), when the roughness of the rail's surface has been introduced into the simulation. The uneven surface has been represented in the FE model in the manner shown in Fig. 7.

It is interesting to note, that the ECAR at $1 \mathrm{~mm}(-0.057)$ was found to be precisely equal to the one determined in the 
case of the FeSi plate, despite different coil characteristics and material properties. Decision, whether this correlation was due to some fundamental physical law, or obtained accidentally, would require additional tests for other sample/coil configurations.

\section{Case of a commercial Electromagnetic Axle Counter}

The postulated transfer function provided an ideal agreement in a full-controlled FeSi plate case, and a good agreement in the narrow-gauge rail track case, where some uncertainty factors (esp. surface roughness) have been allowed.

An ultimate test of a fully industrial situation has been performed, in which the output from a multi-coil sensor was recorded while train wheels moved in its vicinity. The device has been developed and commercialised by VOESTALPINE (Sopot, Poland) under the brand UniAS2. It is supposed to record two key outputs (dynamic resistivity $\mathrm{R}_{\mathrm{d}}$, and the resonance frequency $\omega_{\mathrm{r}}$ ), directly derivable from the amplitude and phase relationship between the current imposed on the circuit and the resulting voltage. The goal of the test was to deduce these key outputs based on the magnetic field computation and the analytical coupling function, and to compare them against experimental ones.

\subsection{Axle Counter: Finite Element Model}

The FEA model created in HyperMesh/ANSYS consisted of 5 million nodes, and 2.6 million SOLID117 elements. Its global structure and meshing details of the skin effect are presented in Figs. 8 and 9.

Similarly to the narrow-gauge rail case, the meshing in the subsurface zone was generated with a progressive bias, so that the outermost layer featured thicknesses of

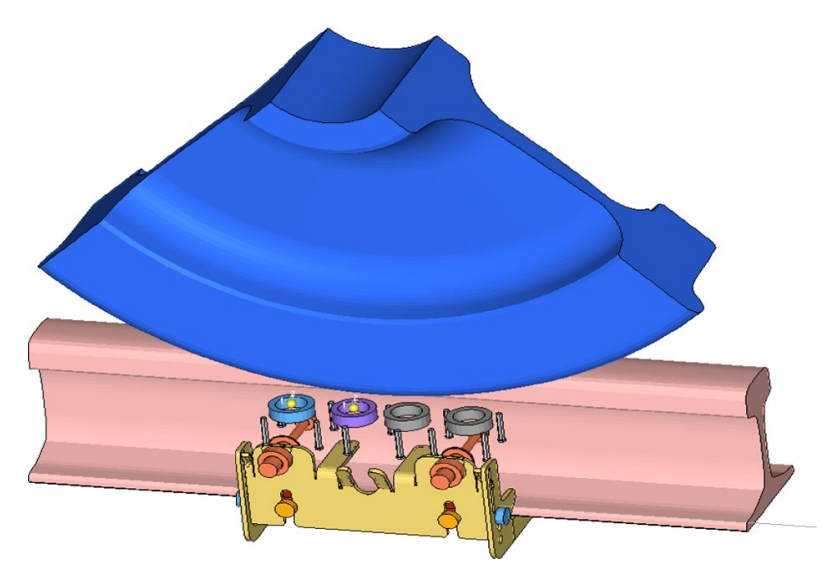

Fig. 8 General view of the eddy-current axle counter (without casing), in the Wheel-Passing state

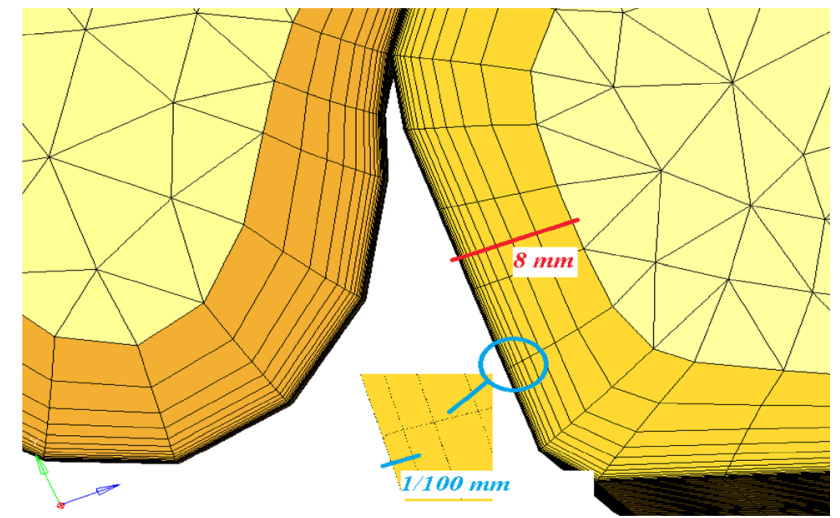

Fig. 9 The detail of the skin-depth meshing of the rail's head and a portion of the wheel

$1 / 100 \mathrm{~mm}$, suitable for correctly reproducing the skin depth effect. Only a $120^{\circ}$ sector of the wheel was modelled for the sake of reduction of the number of elements and associated analysis time. The device was modelled in details, including all the metal plates, screws and bolts, and their surfaces were meshed in a manner adequate to capture the EC influence.

\subsection{Field-Circuit Coupling Function for a Parallel Resonant Circuit}

The postulated coupling function for the parallel RLC circuit has been constructed similarly to the one presented in the case a serial resonant circuit, with some additional assumptions, as follows:

- the effect of wheel velocity is negligible, because of the relatively high operating frequency of the device, exceeding $100 \mathrm{kHz}$, as compared with the frequency of the wheel passages (this could be false for high speed trains, and would require separate study),

- only two extreme cases are considered: "NW"-NoWheel, and "WP"-Wheel-Passing, with the wheel axis situated precisely over the geometrical center of the device,

- the nonlinearity of the rail/wheel material $\mathrm{B}(\mathrm{H})$ curve can be neglected,

- single "effective" L, R and C values can be used, being the arithmetic sum of components (coils, capacitors, resistors) placed on all branches of the parallel circuit,

- the magnetic field amplitude and phase in the neighbouring coils can be averaged and replaced by a single "effective" B composed of a real and an imaginary part.

The derivation of relationship between sensor's output parameters and the magnetic induction starts with the definition of the "dynamic resistance" $R_{d}$ of the parallel RLC 
circuit and its resonant frequency $\omega_{\mathrm{r}}$, which can be generally expressed as:

$R_{d}=\frac{L}{R \cdot C}$,

$\omega_{r}=\frac{1}{\sqrt{C \cdot L}}$.

The effective capacitance $\mathrm{C}$ is a design, constant parameter, while both $\mathrm{L}$ and $\mathrm{R}$ are variables, as already explained in the Sect. 2. The effective resistance of the circuit $\mathrm{R}$ is influenced by the ECs generated in the rail/wheel, so one may write:

$R=R_{0}+R_{E D}$

consequently

$R_{d}=\frac{L}{\left(R_{0}+R_{E D}\right) \cdot C}$,

$R_{d}=\frac{L}{\left(R_{0}+\omega \cdot L_{E D}\right) \cdot C}$.

The effective inductance of the circuit $\mathrm{L}$ is prone to variations due to the "normal" response of the coil to field shielding by metal objects, so

$L=L_{0}+L_{S H}$.

Contrary to the former cases (FeSi plate and narrowgauge track), in this test it is indispensable to perform reference measurements to determine the $\mathrm{L}_{\mathrm{NW}}$ value (i.e. the effective inductance of the coils when NW is present), which includes not only the nominal inductance of the coils, but as well the combined influence of the rail and metal parts of the device itself. Here $\mathrm{L}_{\mathrm{NW}}$ was deduced from the resonance pulsation of the circuit, by a simple transformation of the formula (12):

$L_{N W}=\frac{1}{C \cdot \omega_{r N W}^{2}}$.

The relationships between the magnetic field within the coil's cross-section and the inductance of the coil are proposed in a manner similar to the ones postulated in the serial RL case:

$L_{e d N W}=\frac{B_{i m N W}}{B_{r e N W}} \cdot L_{N W}$,

$L_{\mathrm{WP}}=\frac{B_{r e W P}}{B_{r e N W}} \cdot L_{N W}$,

$L_{e d W P}=\frac{B_{i m W P}}{B_{r e W P}} \cdot L_{\mathrm{WP}}$
Note, that the formulas (18) and (20) are exactly equivalent to (10), while the additional formula (19) constitutes a means of predicting the "Wheel-Passing" inductance of the circuit from the "No-Wheel" inductance and the ratio of calculated magnetic fields.

Knowing the effective $\mathrm{L}$ and its EC related component $\mathrm{L}_{\mathrm{ED}}$ from the formulas above one may now determine the key outputs $R_{d}$ and $\omega_{r}$ of the circuit for the "WheelPassing" state, and comparing them against the reference "No-Wheel" situation. This is fundamental from the point of view of the calibration and interpretation of the signals from the device. Actually, the same universal procedure allows for determining the dynamics of the device for any combination of the rail and wheel, including variable geometry, material data, operating frequency.

Finally, if the entire resonant curve needs to be plotted, one may use the standard formula adequate for parallel RLC circuits:

$\tilde{U}=\tilde{Z} * I=\frac{R_{d}}{1+i \xi} * I$,

where $R_{d}$ is called the dynamic resistivity, and $\xi$ is the detuning factor, representing the difference between the operating frequency $\omega$ and the resonant frequency $\omega_{\mathrm{r}}$ of the circuit

\subsection{Axle Counter: Comparison of Experimental and Modelling Results}

Validation of the presented formulas consisted in calculating the magnetic induction within the coils in both the NW and WP situations, applying the coupling functions and finally comparing the "predicted" and "measured" device dynamics.

The comparison of simulation-based key outputs against averaged data gathered from service is presented below.

Table 3 shows an acceptable agreement between the simulated and service-based data. The dynamics of the dynamic resistance as well as the change of resonant

Table 3 Comparison of key outputs derived from modelling and from experiment in the axle counter test

\begin{tabular}{lc}
\hline Modelling & $\begin{array}{l}\text { Averaged in-service } \\
\text { measurements }\end{array}$ \\
\hline $\begin{array}{ll}\text { Change of dynamic resistance } \mathrm{R}_{\mathrm{d}} \text { during wheel } \\
\text { roll-on }\end{array}$ \\
$-32 \%$ & $-40 \%$ \\
$\begin{array}{l}\text { Change of resonant frequency of the circuit during wheel roll-on } \\
+700 \mathrm{~Hz}\end{array}$ & $+1000 \mathrm{~Hz}$ \\
\hline
\end{tabular}


frequency agree qualitatively, and a fair quantitative correlation is observed despite significant complexity of the device and major sources of uncertainty.

In the commercial continuation of the presented research (detailed results not presented here), both the resonance curve exhibited by the sensor and its maximum output impedance were correctly predicted by the simulation linked the circuit by analytical coupling functions. These results have served as a starting point for a series of modelling of various rail and wheel configurations, and provided a deeper understanding of signal dynamics for three variants of the wheel proximity sensors produced by VOESTALPINE AG.

\section{Conclusions}

In this paper a concise and fairly simple, yet universal methodology for predicting some output characteristics of an EC sensor has been presented. The algorithm in its basic version requires no empirical parameters, relying exclusively on design parameters of the measuring device and the studied object (shape, material, frequency, structure of the electric circuit). The algorithm starts with a standard linear harmonic FEA determining the magnetic induction within the coil(s), followed by the application of coupling functions, linking the field variable to the electrotechnical variables of the circuit. The method finally allows for prediction of key parameter named ECAR, involving the variation of coil's inductance due to the proximity of metal objects.

The method has been validated in three situations of gradually increasing complexity. An ideal agreement between the predicted and experimental results has been achieved in the fully-controlled laboratory tests. A more demanding case of a rail segment with a rough surface produced a very good correlation. Finally, the proposed algorithm passed the fully industrial test, in which a commercial UniAS2 device composed of four coils, placed against a rail and a passing wheel, produced outputs which were successfully predicted by the FE calculation coupled with the coupling functions appropriate for the parallel RLC circuit.

As compared to the fully numerical solution (available to e.g. Flux/Altair software), the presented approach has two advantages. First, the FEA solution required here is linear, whereas the direct computation of the coupling between the magnetic field and the circuit is nonlinear and more difficult to set-up. Second, the analytical passage between the magnetic field and the circuit variables provide a unique insight into the physics behind the device's behaviour and guides changes in the design e.g. by optimal positioning of the sensor.

Proving the validity of the formulas defining ECAR and the capability of predicting sensor's output changes during the wheel passage are a significant, but only partial progress, because of the relative (not absolute) character of the determined relationships. A complete success shall be attained once direct formulas for both $\mathrm{L}_{\mathrm{ED}}(\mathrm{B})$ and $\mathrm{L}_{\mathrm{SH}}(\mathrm{B})$ are found and validated. This is expected to provide a truly universal and practical methodology for predicting, understanding and optimising characteristics of new EC NDT devices and sensors.

Open Access This article is distributed under the terms of the Creative Commons Attribution 4.0 International License (http://creativeco mmons.org/licenses/by/4.0/), which permits unrestricted use, distribution, and reproduction in any medium, provided you give appropriate credit to the original author(s) and the source, provide a link to the Creative Commons license, and indicate if changes were made.

\section{References}

1. Banks, J., Hansford, D.C.: Examination of surface cracks in carbon and manganese steel rails by the eddy current technique. Br. J. NDT 18, 171-174 (1976)

2. Aknin, P., Placko, D., Ayasse, J.B.: Eddy current sensor for the measurement of a lateral displacement. Applications in the railway domain. Sens. Actuators A 31(1-3), 17-23 (1992)

3. Hensel, S., Strauss, T., Marinov, M.: Eddy current sensor based velocity and distance estimation in rail vehicles. IET Sci. Meas. Technol. 9(7), 875-881 (2015)

4. Engelberg, T., Mesch, F.: Eddy current sensor system for noncontact speed and distance measurement of rail vehicles. In: Computers in Railways VII. WIT Press (2000)

5. Geistler, A., Bohringer, F.: Robust velocity measurement for railway applications by fusing eddy current sensor signals. In: Conference Proceedings, 2004 IEEE Intelligent Vehicles Symposium, pp. 664-669

6. Rocha, T.J., Ramos, H.G., Ribeiro, A.L., et al.: Studies to optimize the probe response for velocity induced eddy current testing in aluminium. Measurement 67, 108-115 (2015)

7. Zhua, W., Yina, W., Deweyb, S., et al.: Modeling and experimental study of a multi-frequency electromagnetic sensor system for rail decarburisation measurement. NDT\&E Int. 86, 1-6 (2017)

8. Augustyniak, B., Chmielewski, M., Sablik, M.J., et al.: A new eddy current method for nondestructive testing of creep damage in austenitic boiler tubing. Nondestruct. Test. Eval. 24(1), 121-141 (2009)

9. Kim, T.-O., Lee, G.-S., Kim, H.-Y., Ahn, J.-H.: Modeling of eddy current sensor using geometric and electromagnetic data. J. Mech. Sci. Technol. 21, 465-475 (2007)

10. Enoki, S., Asahi, T., Watanabe, S., et al.: Electromagnetic measurement of the rail displacement by two triangular coils. IEEE Trans. Magn. (2002). https://doi.org/10.1109/TMAG.2002.80229 7

11. Klein, G., Morelli, J., Krause, T.W.: Analytical model of the eddy current response of a drive-receive coil system inside two concentric tubes. NDT\&E Int. 96, 18-25 (2018)

12. Augustyniak, M., Usarek, Z.: Finite element method applied in electromagnetic NDTE: a review. J. Nondestruct. Eval. 35, 39 (2016)

13. Bíró, O., Koczka, G., Preis, K.: Finite element solution of nonlinear eddy current problems with periodic excitation and its industrial applications. Appl. Numer. Math. 79, 3-17 (2014)

14. Gabi, Y., Böttger, D., Straß, B. et al.: Local electromagnetic investigations on electrical steel $\mathrm{FeSi} 3 \%$ via 3MA micromagnetic NDT 
system. In: Proceedings of European Conference on Non-destructive Testing (ECNDT), Gothenburg, December, 2018

15. Tsukerman, I., Dombrovski, V.: Finite-element simulation of time-dependent electromagnetic fields in the end zone of superconducting motors. IEEE Trans. Magn. (2002). https://doi. org/10.1109/20.996323

16. Gabi, Y., Martins, O., Wolter, B., Strass, B.: Combination of electromagnetic measurements and FEM simulations for nondestructive determination of mechanical hardness. AIP Adv. 8, 047502 (2018)
17. Szychta, E., Szychta, L., Kiraga, K.: Analytical model of a rail applied to induction heating of railway turnouts. In: Proceedings of Transport Systems Telematics (TST) 10th Conference, Poland, 20-23 October 2010

18. Żurek, Z.H.: Magnetic monitoring of the fatigue process of the rim material of railway wheel sets. NDT\&E Int. 39, 675-679 (2006)

19. Mehboob, N.: Hysteresis properties of soft magnetic materials. PhD Thesis, Univ. Wien (2012) 